\title{
FIRST RESULTS FROM THE VUV-FEL AT DESY
}

\section{B. Faatz for the DESY VUV-FEL project team, DESY, Notkestr. 85, 22603 Hamburg, Germany.}

\section{Abstract}

The VUV-FEL is an upgrade of the TTF1-FEL, which was in operation until end 2002. During this phase of the project it showed lasing in the wavelength range from 80 $120 \mathrm{~nm}$ and it successfully provided beam for two pilot experiments. For over one year, the machine has been redesigned and upgraded, based on the experience gained during the first phase, to a user facility extending the wavelength range. Commissioning started in February 2004. In this paper, the machine will be described. Important beam parameters, such as emittance, energy spread and bunch profile will be discussed. First results of the characterization of the VUV-FEL will be described in more detail.

\section{MACHINE LAYOUT}

The VUV-FEL at DESY is an upgrade of the TTF1FEL [1,2]. After the TTF1 program was finished end 2002 $[3,4]$, the machine has been extended to a user facility, increasing the energy, doubling the undulator length and completely redesigning the injector [5], based on the experience during the phase 1 operation. The present layout is shown in Fig. 1.

The electrons are generated in a laser-driven photoinjector. The RF-gun has been developed by DESY and commissioned at the Photo Injector Test Stand at DESY Zeuthen (PITZ). It is operated at a gradient of about 40 $\mathrm{MV} / \mathrm{m}$, resulting in an electron beam energy of $4 \mathrm{MeV}$ [6]. Behind the gun, the beam is accelerated to $127 \mathrm{MeV}$ by a first cryo-module developed for TESLA [7], after which it is compressed to achieve the required peak current for lasing. In the following diagnostics section, the beam is matched and the emittance is measured using the four-screen method. In the next two accelerating modules, the beam is further accelerated up to $380 \mathrm{MeV}$, after which a second compressor is installed. Final acceleration results in an energy of up to $750 \mathrm{MeV}$. In the future, an additional module will be installed to reach the final energy of $1 \mathrm{GeV}$. However, at the moment the aim is to produce a laser beam at $30 \mathrm{~nm}$, corresponding to an electron beam energy of $445 \mathrm{MeV}$, resulting in a low energy gain in the last two modules of only $75 \mathrm{MeV}$.

Behind the last accelerating module, additional diagnostics is installed to determine slice parameters of the electron beam. Among them, a transverse deflecting cavity has been installed, combined with a kicker. This combination can kick a single bunch in the direction of an off-axis screen and rotates it in order to resolve the longitudinal bunch profile. In this way, a single bunch out of a bunch train can be analysed while the remaining bunches are availabe to go through a $30 \mathrm{~m}$ long undulator before it reaches the beam dump [8].

Behind the bending magnet towards the beam dump, photon diagnostics has been installed to characterize the photon beam properties. Among them are several apertures to collimate the photon beam, several slits to study the transverse coherence properties, a YAG-screen, a thermopile, a spectrometer and a newly designed microchannel plate detector [9]. A non-interceptive detector is the gas-cell detector, with which position, angle and intensity of the laser beam can be determined.

\section{INJECTOR PERFORMANCE}

In order to guarantee the desired beam quality for lasing, great effort has been put in the design of a new injector. The new gun, tested and commissioned at PITZ, has shown excellent performance [6]. In order to prevent emittance blow-up at low energy, the first accelerating module has been put as close as possible to the gun, leaving only some space for diagnostics and a collimator to reduce the dark current further downstream. The gradient of the first 4 cavities is set to $12 \mathrm{MV} / \mathrm{m}$ for emittance compensation, the second 4 to $20 \mathrm{MV} / \mathrm{m}$ to reach the highest possible energy, resulting in a total energy for the first compression stage of $127 \mathrm{MeV}$. At this energy, extensive emittance measurements have been performed. The projected emittance measured for a $1 \mathrm{nC}$ beam with the four screen method at on-crest acceleration is typically around $2 \mathrm{~mm}$ mrad (for $100 \%$ ) or $1.4 \mathrm{~mm}$ mrad (for $90 \%$ cut). When accelerated off-crest, the projected emittance shows, possibly due to non-closed dispersion, an increased value in the compression plane. The energy spread measured in the compressor is less than $25 \mathrm{keV}$ (see Fig. 2).

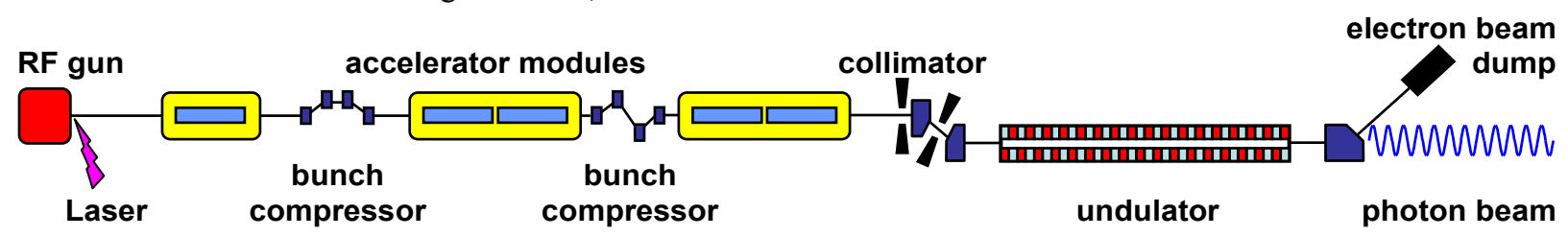

$4 \mathrm{MeV}$

$125 \mathrm{MeV}$

$380 \mathrm{MeV}$

$440 \mathrm{MeV}$

Figure 1: Schematic layout of the VUV-FEL at DESY, Hamburg. 


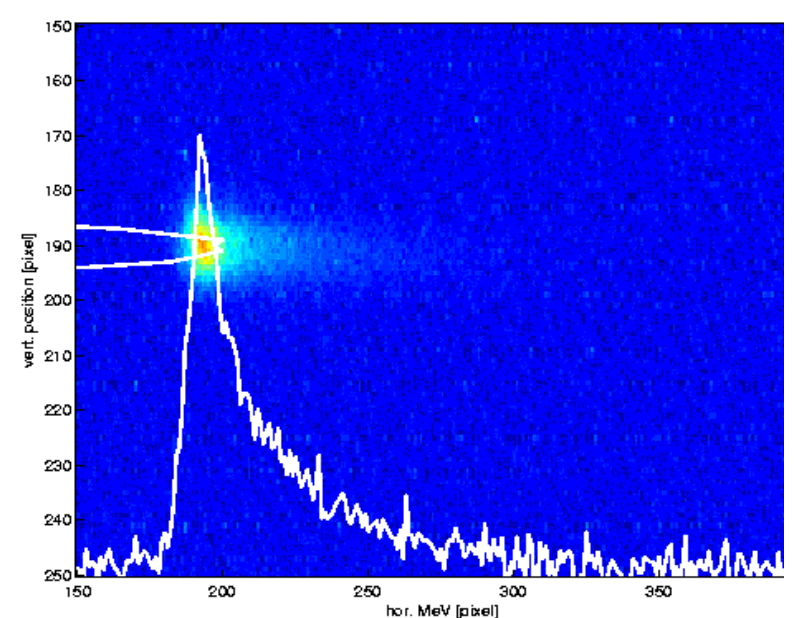

Figure 2: Energy spread measured in the first bunch compressor.

\section{MEASUREMENT OF BUNCH PROFILES}

Most of the electron beam diagnostics used, gives information about the projected properties of the electron beam. In order to resolve the bunch structure, two diagnostics have been implemented into the VUV-FEL, namely Electro-Optical sampling (EOS) and a transverse deflecting cavity 'LOLA'. The latter has been used to characterize the bunch profile during lasing. The setup is shown in Fig. 3. A kicker in front of LOLA deflects the bunch horizontally while RF-field streaks the beam vertically. With this setup, a single bunch out of a train is selected and all others continue towards the undulator.

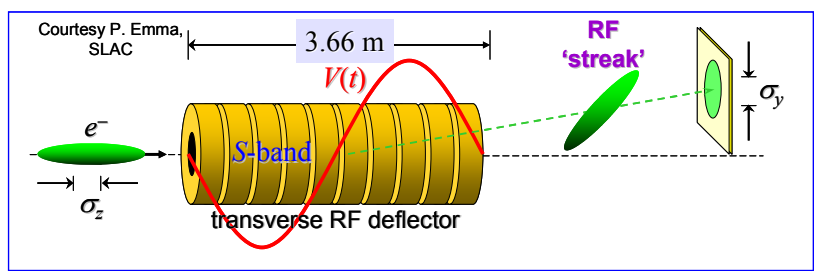

Figure 3: Layout of the LOLA transverse deflecting cavity. A kicker in front of LOLA deflects the beam horizontally and LOLA streaks the beam vertically, thus resolving the longitudinal bunch structure.

A result of such a streak is shown in Fig. 4. The spot of high intensity at the bottom represents the high peak current which leads to lasing. The tail is caused by the $3^{\text {rd }}$ harmonic term in the RF-field at compression, which will be compensated in the future by a third harmonic cavity behind the first accelerating module

\section{Image of LOLA's screen 17ACC7}

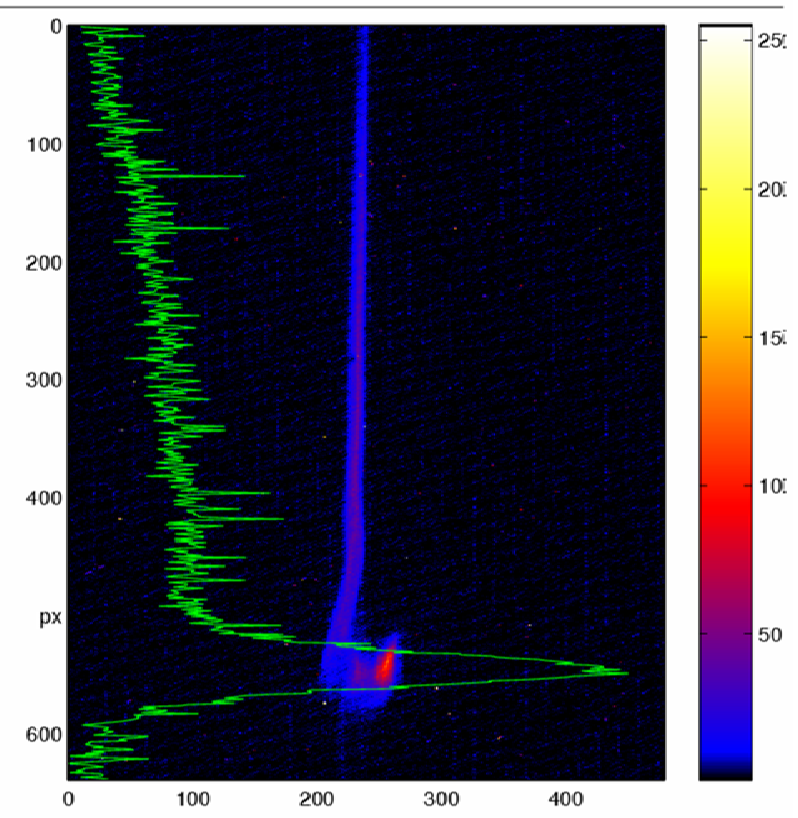

Figure 4: LOLA streak while lasing. Only the small red spot at the bottom of the screen is lasing. The vertical direction is the streak direction in arbitrary units.

\section{FIRST LASING RESULTS}

In order to obtain lasing at $30 \mathrm{~nm}$, only the first bunch compressor at $127 \mathrm{MeV}$ has been used with the first accelerating module between 6 and 10 degrees off-crest and all remaining modules on-crest.

The image of a single FEL pulse with an energy of about $5 \mu \mathrm{J}$ is shown in Fig. 5. The size of the radiation spot, shown here on a 10x10 mm YAG screen, is about $3 \mathrm{~mm}$ which is approximately expected at this distance. The exact size of the radiation field expected at this position depends on the effective location of the source along the

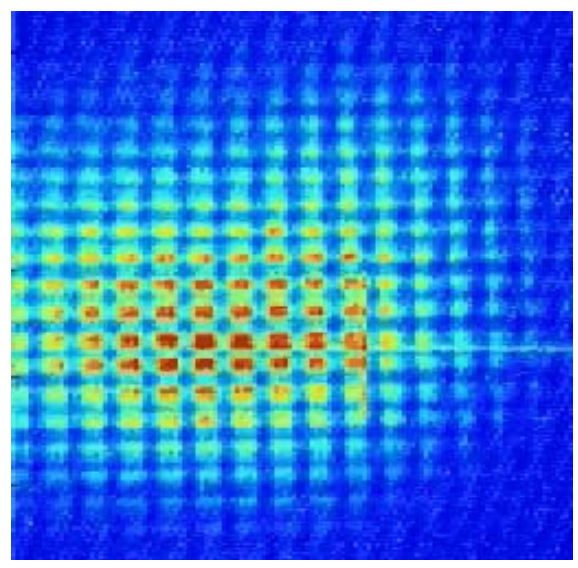

Figure 5: The FEL beam image of a single bunch shown on a YAG crystal. The structure is due to the mesh in front used to reflect the light to a MCP detector. 
undulator, which is not yet known exactly at this time. Three single shot spectral distributions are shown in Fig. 6 (top figure). For comparison, the spectra expected from start to end simulations are shown also shown in Fig. 6 (bottom figure).
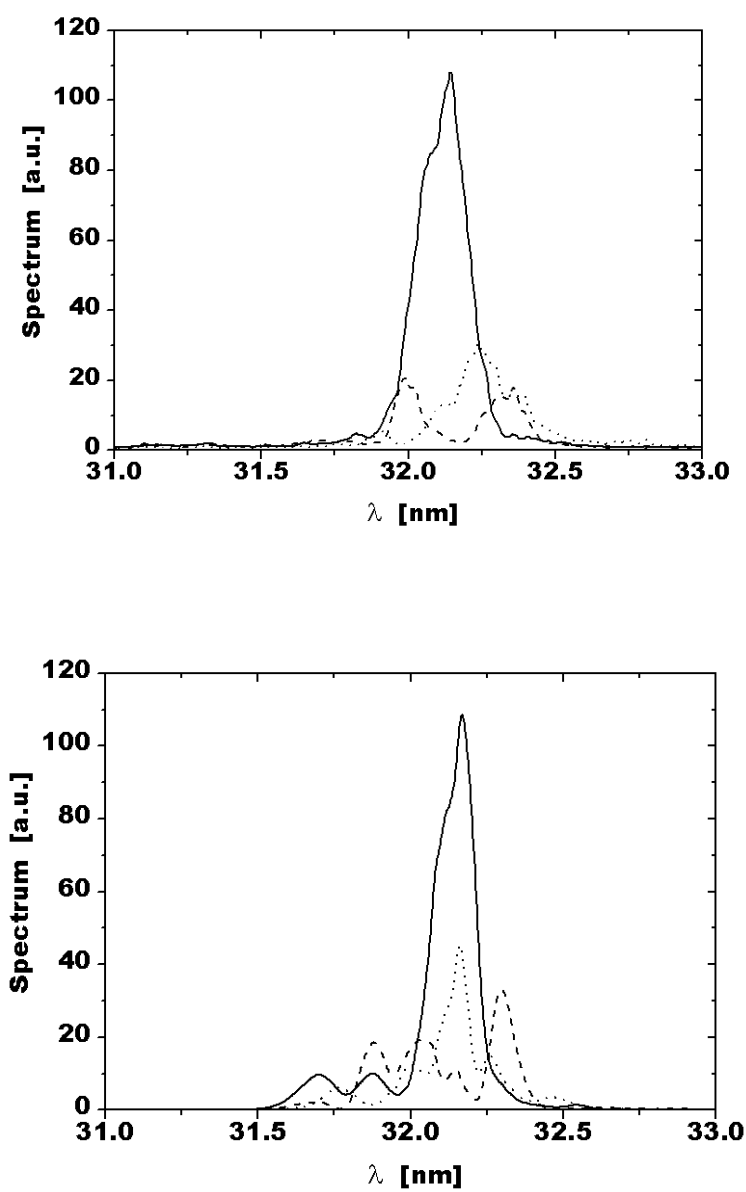

Figure 6: Three single-shot spectra of FEL radiation pulses measured at the VUV-FEL (top), represented by a solid, dashed and dotted lines. Bottom: Simulation of the spectral structure of the VUV-FEL operating in the exponential regime [10].

Figure 7 shows the measured energies at $32 \mathrm{~nm}$ wavelength of many successive radiation pulses. A large fluctuation is seen which is well understood since in a SASE FEL the gain process starts from shot noise. Theoretically, the radiation pulse energy should fluctuate according to a Gamma distribution provided the FEL gain process is in the regime of exponential growth $[11,12]$. The parameter $M$ is the inverse of the normalized variance of the mean photon beam energy $E$. It defines the number of optical modes in the radiation pulse and provides a relationship between the average number of spikes in the single-pulse wavelength spectra and the fluctuations of pulse energy. The measured histogram in the lower part of Figure 7 fits nicely the Gamma distribution with $M=4.1$.
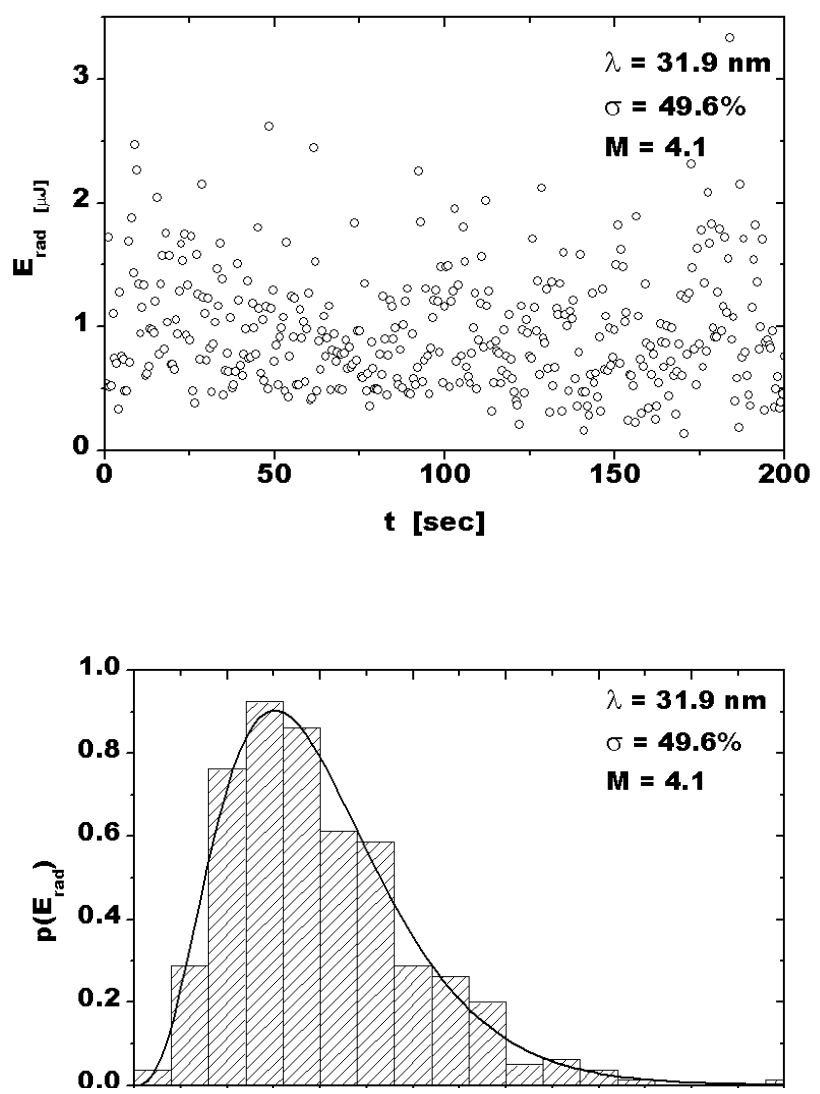

Figure 7: Measured FEL-pulse energies for many successive pulses in the high-gain regime. Top: pulse energy vs. time. Bottom: Measured probability distribution of the radiation energy (histogram). The solid curve represents the Gamma distribution $\mathrm{p}(\mathrm{E})$ for $M=4.1$ which is calculated from the variance of pulse energy fluctuations illustrated in the upper part of the figure. The measurement confirms that the VUV-FEL is operating in the exponential regime and is a source of completely chaotic polarized radiation $[11,12]$, with $M$ being the total number of optical modes in the pulse.

Even though there is still uncertainty concerning the electron beam parameters at the undulator entrance, the results presented here agree well with simulations performed. The parameters obtained by start-to-end simulations at the undulator entrance are given in Table 1, together with a summary of the photon beam parameters. 
Table 1: VUV-FEL Parameters from Start-to-End Simulations

\begin{tabular}{|l|l|l|}
\hline Electron Parameter & Unit & \\
\hline Energy spread & $\mathrm{keV}$ & 300 \\
\hline Emittance & $\mathrm{mm} \mathrm{mrad}$ & 3 \\
\hline Peak Current & $\mathrm{kA}$ & 2 \\
\hline Bunch length (lasing) & $\mathrm{fs}$ & 50 \\
\hline Photon Parameters & & \\
\hline Pulse energy & $\mu \mathrm{J}$ & 5 \\
\hline Number of photons & & $8.10^{11}$ \\
\hline Gain & & $10^{6}$ \\
\hline
\end{tabular}

The $5 \mu \mathrm{J}$ power mentioned before indicates that we are about an order of magnitude away from saturation. Note that compared to the emittance measured in the injector, these numbers indicate a growth along the entire machine of about $50 \%$. The increased energy spread at the undulator entrance is caused by compression. Since the leading spike in the electron beam distribution is approximately Gaussian, the photon pulse length is close to a factor of 2 smaller, resulting in a 20 to 25 fs long laser pulse. With this pulse length consisting of 4 spikes, one can deduce that the cooperation length is 6 fs or $2 \mu \mathrm{m}$ and the gain length is approximately $2 \mathrm{~m}$.

\section{SUMMARY}

The VUV-FEL has been commissioned to large extent. Lasing has been achieved at the design wavelength of 30 $\mathrm{nm}$ with all the predicted properties. With most of the sub-systems working, the short term goal is to reach saturation, commission the user beam lines, which has already started and, within the next few months, supply $30 \mathrm{~nm}$ high-intensity radiation to users. In addition to user dedicated beam time, the $R \& D$ program will continue and the wavelength range will be extended.

\section{REFERENCES}

[1] A VUV Free Electron Laser at the TESLA Test Facility at DESY. Conceptual Design Report, DESY, TESLA-FEL 95-03 (1995).

[2] The TTF FEL team, SASE FEL at the TESLA Test Facility, Phase 2, DESY, TESLA FEL 2002-01 (2002).

[3] V. Ayvazyan et al., Generation of GW radiation pulses from a VUV free-electron laser operating in the femtosecond regime, Phys. Rev. Lett. 88, 104802 (2002).

[4] v. Ayvazyan et al., A new powerful source for coherent VUV radiation: Demonstration of exponential growth and saturation at the TTF freeelectron laser, Eur. Phys. J. D20, 149 (2002).

[5] K. Floettmann, T. Limberg, Ph. Piot, Generation of ultra-short electron bunches by cancellation of nonlinear distortions in the longitudinal phase space, TESLA-FEL report 2001-06, DESY, Germany.

[6] R.J. Bakker et al., Nucl. Instrum. Methods A507 (2003) 210.

[7] H. Weise, Superconducting RF Structures - Test Facilites and Results, Proc. 2003 Part. Acc. Conf. p. 673 (2003).

[8] J. Pflüger, Undulators for SASE FELs, Nucl. Instrum. and Methods A445, 366 (2000).

[9] R. Treusch et al., Photon diagnostics on the VUVFEL at DESY: First lasing around $100 \mathrm{~nm}, \mathrm{Nucl}$. Instrum. and Methods A467-468, 30 (2001).

[10] E.L. Saldin, E.A. Schneidmiller, \& M.V. Yurkov, Expected properties of the radiation from VUV-FEL at DESY (femtosecond mode of operation), DESY, TESLA-FEL 2004-06 (2004).

[11] E.L. Saldin, E.A. Schneidmiller, \& M.V. Yurkov, The Physics of Free Electron Lasers, SpringerVerlag, Berlin (1999).

[12] E.L. Saldin, E.A. Schneidmiller, \& M.V. Yurkov, Statistical properties of radiation from VUV and Xray free electron lasers, Opt. Commun. 148, 383 (1998). 